\title{
Statistical analysis of SEM image noise
}

\author{
C. Mansilla, V. Ocelík \& J. Th. M. De Hosson \\ Materials innovation institute M2i, Department of Applied Physics, \\ University of Groningen, The Netherlands
}

\begin{abstract}
The stress-relaxation technique is one of the most popular approaches to measure macroscopic residual stresses in different kinds of materials. Recently, by using a dual beam microscope (Scanning Electron Microscope (SEM) and Focused Ion Beam (FIB) in one chamber), a new option of detection of internal stresses on a microscopic scale appeared. FIB is used to release local strain induced by internal stresses and Digital Image Correlation (DIC) of SEM images collected before and after stress release is applied to quantify strain release at the surface.

However, the combination of DIC and SEM brings some new important aspects in the analysis of noise generated by the special way of building an SEM image. Because the magnitude of the strain is quite often very close to the noise level, it is very important to test each SEM column which is used in these measurements. It is necessary to study conditions for minimization and randomization of the noise generated during SEM image collection by instabilities inside electron column and sample chamber as well. In the present work, the methods for such testing will be given for a Philips XL30 ESEM.

Keywords: residual stress, scanning electron microscopy, focus ion beam, digital image correlation, self-correlation image, slit milling.
\end{abstract}

\section{Introduction}

Residual stresses can be defined as the stresses that persist after the extrinsic cause of deformation (external forces, heat gradients, etc.) has been removed [1]. They are present in almost all materials, and arise whenever inelastic processes occur, like plastic deformation or thermal treatments. These stresses influence the lifetime of products in service, for instance by accelerating crack growth in the material. As a consequence exact knowledge and control of residual stresses are of high practical relevance. 
The traditional methods available for measuring internal stresses can be divided into destructive and non-destructive. The most prominent and versatile non-destructive method is X-ray diffraction [2]. However, it requires crystalline samples. Common destructive methods are curvature measurement [3], nanoindentation [4], hole drilling [5], etc. In addition, most of them determine the mean stress in large volumes or areas.

The so-called slit-milling method combines the ion milling and imaging capabilities provided by a dual beam microscope. Therefore SEM images are taken of the specimen i.e. before and after a fine slit has been made by ion milling. Digital Image Correlation (DIC) $[6,7]$ is used to measure the displacements due to stress release, and the residual stress is calculated from linear elasticity theory. As the method is not based on diffraction technique it can be applied on almost any solid state material including amorphous layers, thin coatings, polymers, etc. In addition, due to the small size of the slit, it may provide also local information.

Several authors [8-10] have previously used SEM image correlation to measure stresses on the top of a coating by relaxation displacement measurement after FIB milling. DIC is an image-based method that consists in dividing the images to be compared in smaller sub-regions, called 'facets' [6]. Each facet must contain enough features that it can unambiguously correlate with the same sub region in the next image. Then, these facets are compared by matching and when the highest correlation is found the displacements of the center of these sub-regions can be obtained. These displacements can be related to the strain induced by internal stress.

The displacements are often in the sub-pixel size, but they can be accurately determined by DIC. However, the displacements due to instabilities of the SEM equipment could be in the same range than the displacements caused by the stress release. Therefore, an evaluation of the SEM stability is crucial for this type of application. The aim of this work is the evaluation of the stability of a XL30 ESEM from Philips, and the development of the appropriate statistical tools to characterize the nature of the observed instabilities.

\section{Experimental}

\subsection{Mechanism of SEM image acquisition: introduction of general image parameters}

Since different scanning electron microscopes can be studied, a short description of imaging process and parameters is appropriate. When scanning a specimen surface with a finely focused electron beam (one of only several nanometers), secondary electrons are emitted from each point of the scanning. These are converted into an electrical signal, amplified, and finally displayed as an image on the screen or file. During scanning different parameters can be selected depending on the specimen surface or the quality of the desired image. These parameters are summarized in Figure 1, which shows the schematics of an image formation in an SEM. A focused beam with an energy $E$, small spot size (smaller 
than the pixel size) and a fixed working distance is scanned horizontally pixel by pixel (resolution) with a fixed time per pixel (dwell time) until the end of the line (horizontal image size). Working distance is the distance between the last lens in the e-column and the surface of the specimen. Then, the beam quickly returns to the beginning of the next line and a line scan is performed again. The number of lines will define the vertical image size.

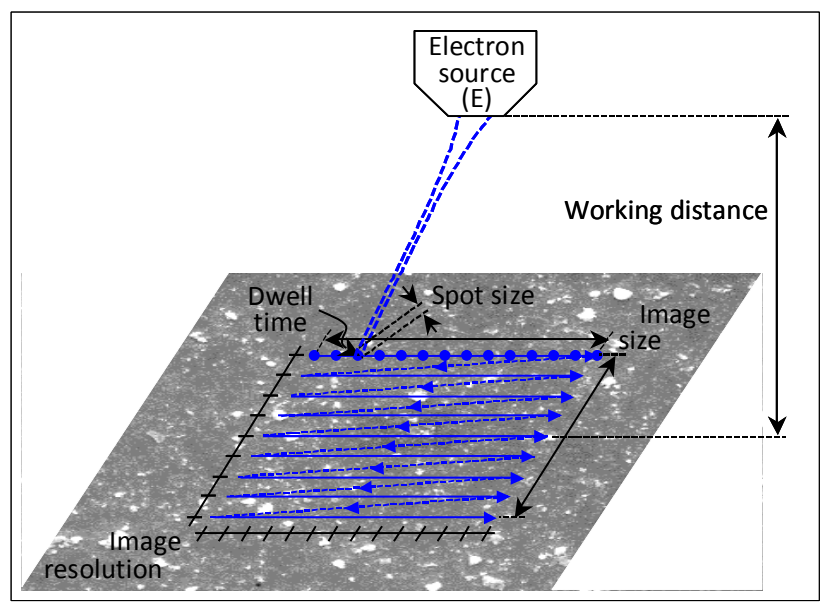

Figure 1: $\quad$ Schematics of the formation of an image in an SEM equipment.

\subsection{Description of the study}

The stability of SEM images is evaluated by DIC measuring the error (noise) between two images taken under the same conditions, at the same place and with a certain delay time between them. Thus, the study starts with the acquisition of a first image of the area to be analyzed. Afterwards, the sample holder is moved away and returned back, and then a second image of the same area is collected. A time delay of 2-3 min between consecutive images was used. This procedure was repeated for the different imaging conditions shown in Table 1 in order to study how these conditions may influence the stability of the microscope image.

During DIC analysis, the images were divided in small regions called facets. In all cases of our analysis, a facet size of $43 \times 43$ pixels, with a step between the centers of neighbor facets of 21 pixels was selected. These dimensions were chosen considering the contrast of each image and to avoid errors in the DIC.

Since DIC is an image-based method, a high contrast on the surface is required in order to get good results. Different surface decoration techniques have been found in literature depending on the studied surface area and the homogeneity of the decoration (pattern) [11]. Deposition of yttria-stabilizedzirconia (YSZ) nanoparticles is a good method because it can be applied rapidly on large surface areas [9]. Therefore, YSZ nanoparticles were deposited on the surface from a $40 \%$ aqueous ethanol suspension $\left(\sim 30 \mathrm{mg} / \mathrm{cm}^{3}\right)$ and dried at room temperature before the measurements. An ultrasonic bath was used for 30 min to 
break up the large YSZ particle agglomerates in the suspension prior to the deposition.

\subsection{Equipment and parameters under study}

Different experiments by changing the imaging parameters have been performed in a field emission XL30 ESEM scanning electron microscope from Philips. Table 1 shows the different imaging conditions under study.

Table 1: $\quad$ Imaging conditions studied for Philips ESEM XL30.

\begin{tabular}{|c|c|c|c|c|c|c|}
\hline Cond. & $\begin{array}{c}\text { Beam Energy } \\
(\mathbf{k V})\end{array}$ & $\begin{array}{c}\text { Spot Size } \\
(\mathbf{u n i t s})\end{array}$ & $\begin{array}{c}\text { WD } \\
(\mathbf{m m})\end{array}$ & $\begin{array}{c}\text { Resolution } \\
(\mathbf{p x})\end{array}$ & $\begin{array}{c}\text { Pixel size } \\
(\mathbf{n m})\end{array}$ & $\begin{array}{c}\text { Dwell time } \\
(\boldsymbol{\mu} \mathbf{s})\end{array}$ \\
\hline P1 & 10 & 5 & 10 & $1296 \times 968$ & $\mathbf{2 3 0 . 0}$ & 41 \\
\hline P2 & $\mathbf{1 0}$ & $\mathbf{5}$ & $\mathbf{1 0}$ & $\mathbf{1 2 9 6} \times \mathbf{9 6 8}$ & $\mathbf{2 3 . 3}$ & $\mathbf{4 1}$ \\
\hline P3 & 10 & 5 & 10 & $1296 \times 968$ & $\mathbf{1 1 . 6 5}$ & 41 \\
\hline P4 & 10 & 5 & 10 & $\mathbf{1 9 3 6} \times \mathbf{1 4 5 2}$ & $\mathbf{1 5 . 6}$ & $\mathbf{2 8}$ \\
\hline P5 & 10 & 5 & 10 & $1296 \times 968$ & 23.3 & $\mathbf{6 2}$ \\
\hline P6 & 10 & 5 & $\mathbf{5}$ & $1296 \times 968$ & 23.3 & 41 \\
\hline P7 & 10 & $\mathbf{3}$ & 10 & $1296 \times 968$ & 23.3 & 41 \\
\hline P8 & $\mathbf{5}$ & 5 & 10 & $1296 \times 968$ & 23.3 & 41 \\
\hline P9 & $\mathbf{3}$ & 5 & 10 & $1296 \times 968$ & 23.3 & 41 \\
\hline
\end{tabular}

The modified parameters were: electron beam energy, spot size, working distance (WD), image resolution, pixel size and dwell time. Condition P2 is considered as reference, and the modified parameters are highlighted in bold in each case. It is worth mentioning that this microscope works under a single scan mode, so no integration of subsequent images was tested.

\subsection{Description of the statistics performed to analyze the stability}

Three different approaches have been followed to evaluate the stability of the microscope. The first one is more 'generic', and aims at evaluating the overall degree of noise in the measurements. The other two aim at identifying an order in the noise. The first of them is designed to find patterns in rows and columns, as expected from the way of forming an SEM image (see Fig. 1). In the second, any type of ordered distribution is visualized.

Thus, the instability of the SEM image is expressed as a value of standard deviation $(S D)$ of displacements of the centers of all facets between subsequent 
recorded images calculated for both directions: $\mathrm{x}$ - horizontal and y-vertical $\left(S D_{x}\right.$, $\left.S D_{y}\right)$.

To evaluate the randomness of noise found during our DIC evaluation, we use two strategies. In the first one, we calculate the ratio $(R)$ between the average of the standard deviations ( $\overline{S D}$ ) of the displacements in image columns $\left(\overline{S D}^{\text {cols }}\right)$ and lines $\left(\overline{S D}^{\text {rows }}\right)$ for both directions. The average of the standard deviation $(\overline{S D})$ of the displacements by columns is defined by:

$$
\overline{S D}^{\text {cols }}=\frac{1}{m} \sum_{j=1}^{m} S D_{j}=\frac{1}{m} \sum_{j=1}^{m} \sqrt{\frac{1}{n} \sum_{i=1}^{n}\left(z_{i}(j)-\overline{z_{i}(j)}\right)^{2}}
$$

being $n$ and $m$ the number of columns and lines, respectively. The standard deviation $(\overline{S D})$ of the displacements in the line is:

$$
\overline{S D}^{\text {rows }}=\frac{1}{n} \sum_{i=1}^{n} S D_{i}=\frac{1}{n} \sum_{i=1}^{n} \sqrt{\frac{1}{m} \sum_{j=1}^{m}\left(z_{j}(i)-\overline{z_{j}(i)}\right)^{2}}
$$

Thus,

$$
\text { Ratio }(R)=\frac{\overline{S D}^{\text {cols }}}{\overline{S D}^{\text {rows }}}
$$

This expression will make possible to evaluate the presence of correlation in one direction with respect to the other. In case of square images, $R$ has an easy interpretation:

- If $\mathrm{R}<1$, higher correlation in columns than in rows.

- If $\mathrm{R}>1$, smaller correlation in columns than in rows.

- If $\mathrm{R}=1$, it does not give any information.

A second way to evaluate the presence of correlation and periodicity is the self-correlation of the displacement image matrix [12]. Self-correlation image is defined by:

$$
G\left(k_{1}, k_{2}\right)=\sum_{x, y} f(x, y) f\left(x+k_{1}, y+k_{2}\right)
$$

where $f(x, y)$ is the image matrix. This equation compares the image and the same image shifted over a distance $k_{1}$ and $k_{2}$ in the $x$ and $y$ axis with respect to the center of the image. The result is a new image $G\left(k_{1}, k_{2}\right)$, which is a measure of how different the two images are. The more equal the image and the shifted image are, the higher the value of the self-correlation is. The periodicity in the original image will be shown as a periodic pattern in the self-correlation image [12]. In self-correlation, the highest value is obtained at the center of the image (i.e. where $k_{1}$ and $k_{2}$ are zero).

The self-correlation analysis helps not only to identify the presence of order, but also to distinguish its kind. The standard deviation has been calculated for each self-correlation image $\left(S D^{s c}\right)$, in order to somehow quantify the degree of patterning. Thus, it is expected that images with low degree of order will show lower values of this parameter than highly ordered images; the former ones 
should show a homogenous distribution, while in the latter case the selfcorrelation images will include regions with positive and negative correlation.

\section{Results and discussion}

Table 2 shows all above mentioned statistical parameters calculated for all different imaging conditions for the ESEM XL-30 from Philips.

Table 2: $\quad$ Statistical parameters obtained for SEM images collected by Philips ESEM XL-30.

\begin{tabular}{|c|c|c|c|c|c|c|c|c|c|c|}
\hline Cond. & $\begin{array}{l}\mathrm{SD}_{\mathbf{x}} \\
(\mathbf{p x})\end{array}$ & $\begin{array}{l}\mathrm{SD}_{\mathbf{y}} \\
(\mathbf{p x})\end{array}$ & $\begin{array}{r}\overline{\mathrm{SD}}_{\mathbf{x}}^{\text {cols }} \\
(\mathbf{p x})\end{array}$ & $\begin{array}{c}\overline{\mathrm{SD}}_{\mathbf{x}}^{\text {rows }} \\
(\mathbf{p x})\end{array}$ & $\mathbf{R}_{\mathbf{x}}$ & $\begin{array}{r}\overline{\mathrm{SD}}_{\mathrm{y}}^{\text {cols }} \\
\text { (px) }\end{array}$ & $\begin{array}{c}\overline{\mathrm{SD}}_{\mathbf{y}}^{\text {rows }} \\
(\mathbf{p x})\end{array}$ & $\mathbf{R}_{\mathbf{y}}$ & $\mathrm{SD}_{\mathrm{x}}^{\mathrm{sc}}$ & $\mathrm{SD}_{\mathrm{y}}^{\mathrm{sc}}$ \\
\hline $\mathrm{P} 1$ & 0.025 & 0.041 & 12.6 & 66.6 & 0.19 & 7.69 & 8.97 & 0.86 & 201 & 71.1 \\
\hline P2 & 0.059 & 0.042 & 1.07 & 1.08 & 0.99 & 0.87 & 0.74 & 1.18 & 30.1 & 22.0 \\
\hline P3 & 0.155 & 0.490 & 0.852 & 1.28 & 0.67 & 5.57 & 0.87 & 6.39 & 33.4 & 58.8 \\
\hline P4 & 0.090 & 0.091 & 2.09 & 1.31 & 1.60 & 2.06 & 1.25 & 1.65 & 9.97 & 21.1 \\
\hline P5 & 0.074 & 0.219 & 1.13 & 0.94 & 1.21 & 5.03 & 0.99 & 5.10 & 206 & 101 \\
\hline P6 & 0.074 & 0.103 & 1.65 & 1.34 & 1.26 & 2.30 & 1.38 & 1.67 & 31.8 & 29.6 \\
\hline P7 & 0.079 & 0.210 & 1.17 & 1.72 & 0.68 & 4.89 & 1.20 & 4.06 & 18.3 & 106 \\
\hline P8 & 0.124 & 0.268 & 1.94 & 2.81 & 0.69 & 5.92 & 2.27 & 2.61 & 105 & 66.5 \\
\hline P9 & 0.048 & 0.121 & 1.09 & 0.87 & 1.26 & 2.85 & 0.77 & 3.71 & 8.77 & 36.9 \\
\hline
\end{tabular}

Pixel size was modified between conditions P1 to P4 by changing the image size and the image resolution (cf. Table 1). For these conditions, the dependence of the $S D_{x}$ and $S D_{y}$ on the pixel size is shown on Figure 2. Since the pixel size is not constant, there are two non-equivalent possibilities to evaluate the $S D$ (in px and $\mathrm{nm}$ ), and both are included in this figure. It can be seen that $S D_{x}$ and $S D_{y}$ expressed in pixels increase when pixel size is reduced as it can be expected. However, for practical applications we are interested in displacements expressed in nanometers, which are depicted in Figure 2b. Despite the low values of SDs in pixels observed for $\mathrm{P} 1$, this condition shows the highest value in $\mathrm{nm}$ as a consequence of the large pixel size used in this case. On the contrary, P3 has the smallest pixel size, but the $S D$ in $\mathrm{nm}$ is still high due to the high $S D$ in pixel. The intermediate cases (P2 and P4) are the best (i.e. lowest values of $S D$ in $\mathrm{nm}$ ), since they were acquired using a magnification high enough to reach a reasonably small pixel size without large instabilities (i.e. high values of $S D$ in pixels). It is also worth mentioning that an increase of the resolution (P4 vs. P2) does not lead to any improvement in image stability. 


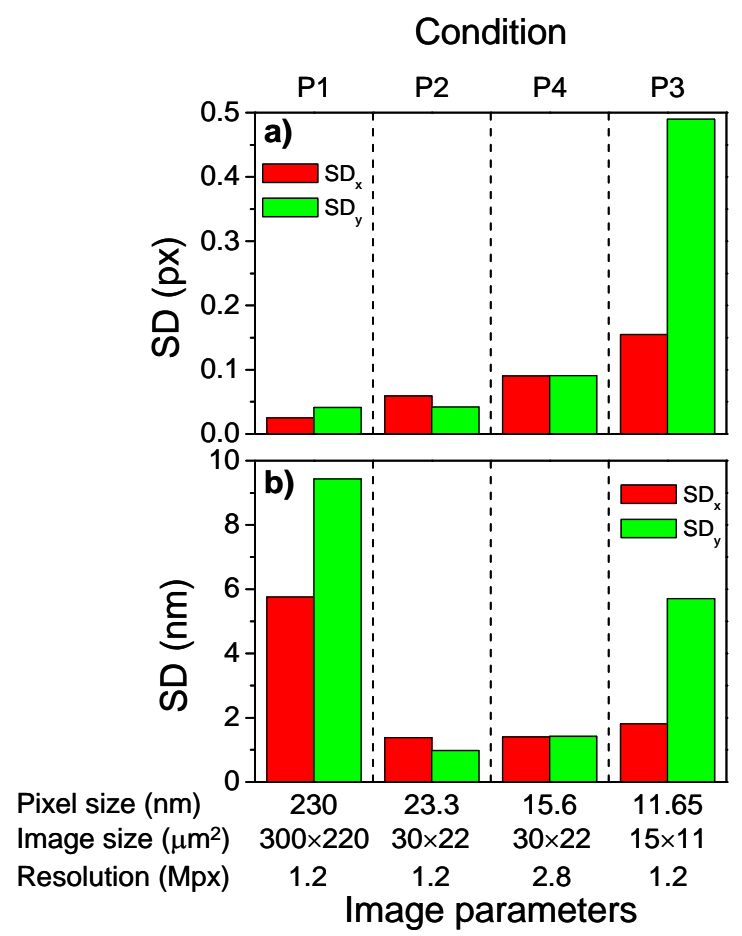

Figure 2: $\quad$ Influence of the pixel size on the $S D_{x}$ and $S D_{y}$ from conditions P1 to P4. Top: $S D$ expressed in pixels. Bottom: $S D$ expressed in $\mathrm{nm}$. The imaging parameters and labels used in each condition are included in the bottom and top $x$ axis, respectively.

In general this microscope seems to be quite unstable and it was difficult to find clear relations between imaging conditions and statistical parameters. However, it is possible to illustrate relationships between observed instabilities on images and statistical parameters. To do that, we will focus on conditions with the same pixel size, in order to facilitate the interpretation of all the statistical parameters (all calculations performed in pixels). Therefore, conditions P1, P3 and P4 will be not considered from now.

Figures 3 and 4 show the overlay of horizontal (top rows) and the vertical (bottom rows) displacements obtained by DIC for two subsequent images collected at the conditions P2, P5, P6, P7, P8 and P9. In general, the ranges of vertical displacements are larger than the corresponding ranges of horizontal displacements in all cases, except P2. This trend is confirmed in Table 2, since in these situations $S D_{y}$ is larger than $S D_{x}$.

However, there is no direct relationship between the displacement range and the values of SD. For instance, P2, P5 and P9 show similar displacement scale bars in $x$ direction (ca. $8 \mathrm{~nm}$ ), but very different values of $S D_{x}$. In $y$ direction, P6 and P9 also show an equivalent displacement bar (ca. $18 \mathrm{~nm}$ ) and different 


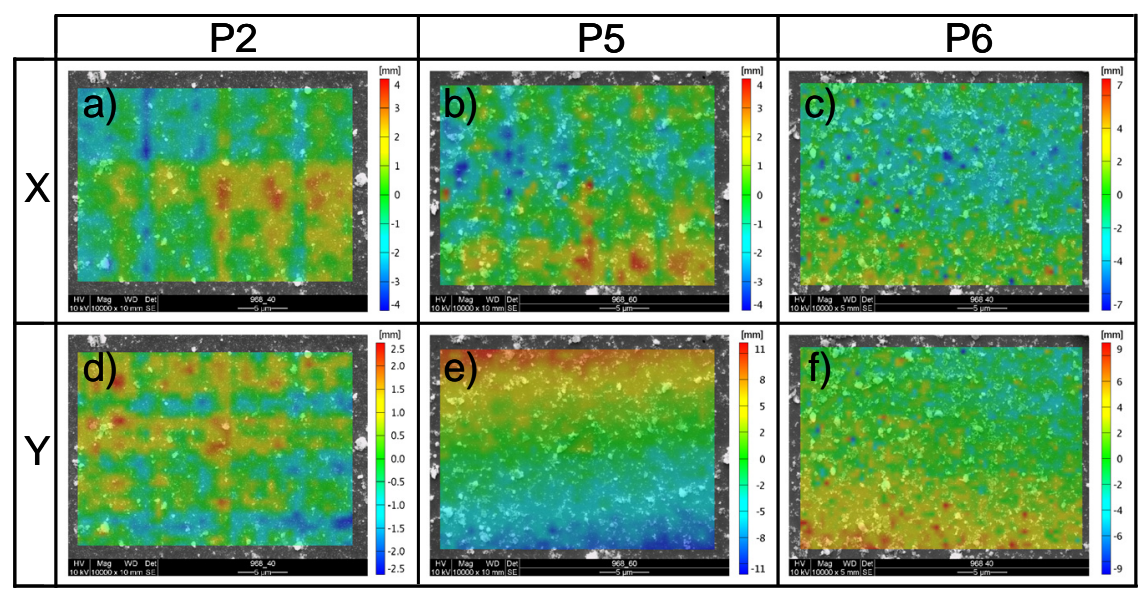

Figure 3: Images of displacement in $x$ (top) and in $y$ (bottom) directions obtained by DIC from conditions P2, P5 and P6. The units of the displacement color bars are nm.

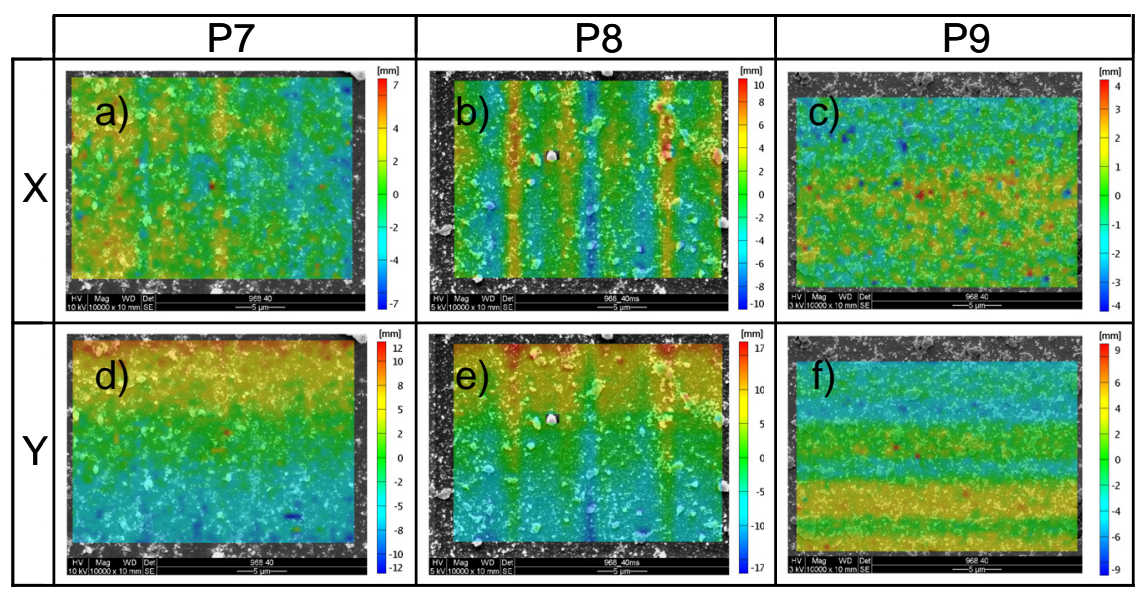

Figure 4: Images of displacement in $x$ (top) and in $y$ (bottom) directions obtained by DIC from conditions P7, P8 and P9. The units of the displacement color bars are nm.

values of $S D_{y}$. Therefore, it can be stated that $S D$ is not merely an indicator of the range of the displacements, but also informs about its real distribution.

Nevertheless, different types of distributions can be distinguished for the conditions presented in Figures 3 and 4, where images with random or ordered distributions (with horizontal, vertical or even both types of bands) can be found. Therefore, it is important to use appropriate statistical parameters which reveal an order present in the displacement images. 
To evaluate the presence of order in columns and lines, the parameter $R$ has been defined (see eq. (3)). Since in this case the images are rectangular, the value of $R \sim 1.2$ indicates disordered structures (cf. conditions P6-x and P9-x in Figs. 3c and 4c). Thus, values of $R<1.2$ informs us of the presence of vertical bands (e.g. condition P8-x in Fig. 4b). On the contrary, a value of $R>1.2$ is associated to horizontal bands (e.g. see condition P9-y in Fig. 4f).

Considering this parameter, the most stable condition appears to be P6 which shows a random noise distribution in $x$ with value of $R_{x}$ close to 1.2 (see Table 2) and smooth horizontal bands in $y$. For the rest of conditions, in general it can be seen that displacements in $x$ are grouped in vertical bands, except in case of P5 where both types of bands are obtained. On the contrary, displacements in $y$ are mostly grouped in horizontal bands, except P2 which shows both of them. It is worth mentioning that for the conditions P2-y (Fig. 3d) and P5- $x$ (Fig. 3b) the parameter $R$ does not provide information about any presence of ordering, although bands are present in the images. In these cases $R$ is close to 1.2 , the value of a random distribution. However, the images of the displacements do not show a random distribution, but a similar order in both directions, i.e. presence of both horizontal and vertical bands with the same weight. Therefore, in order to further clarify these cases, it is useful to use the self-correlation images to evaluate the order of bands obtained by DIC.

Figures 5 and 6 depict the self-correlation images of the DIC images displayed in Figures 3 and 4, respectively. In this case, the periodicity present in the DIC displacement images appears as periodic patterns. In general, selfcorrelation images show the same information revealed by $R$. However, it can distinguish random distribution (see Figs $5 c$ and $6 c$ ) from ordered distributions (see Fig. 5b and 5d). In particular, this latter example reflects clearly the

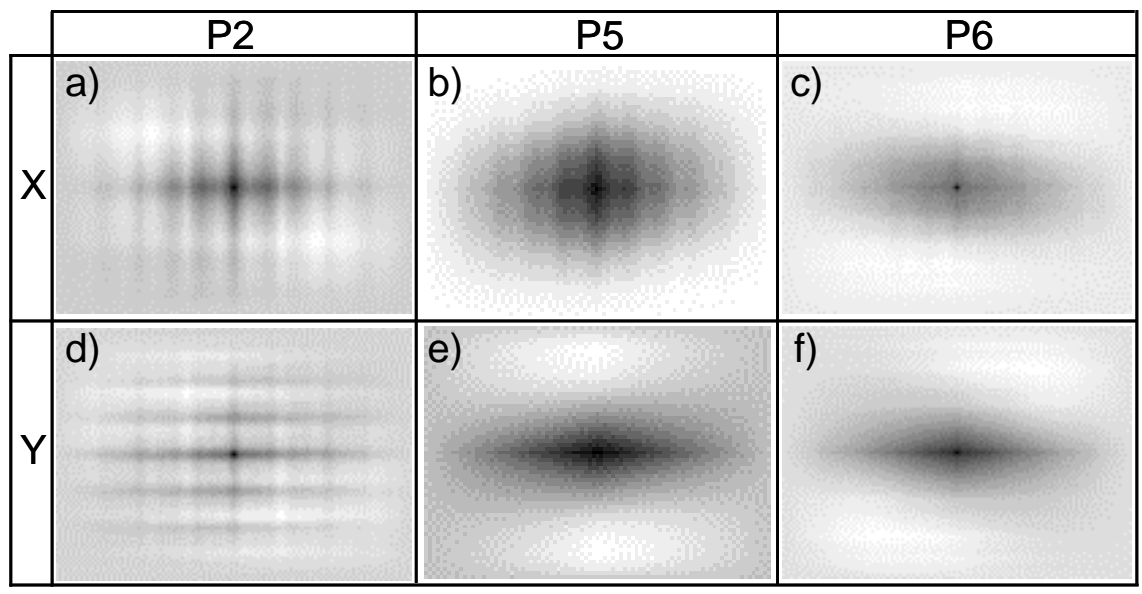

Figure 5: Images of self-correlation corresponding to images of displacement in $x$ (top) and in $y$ (bottom) directions from conditions P2, P5 and P6 shown in Figure 3. 


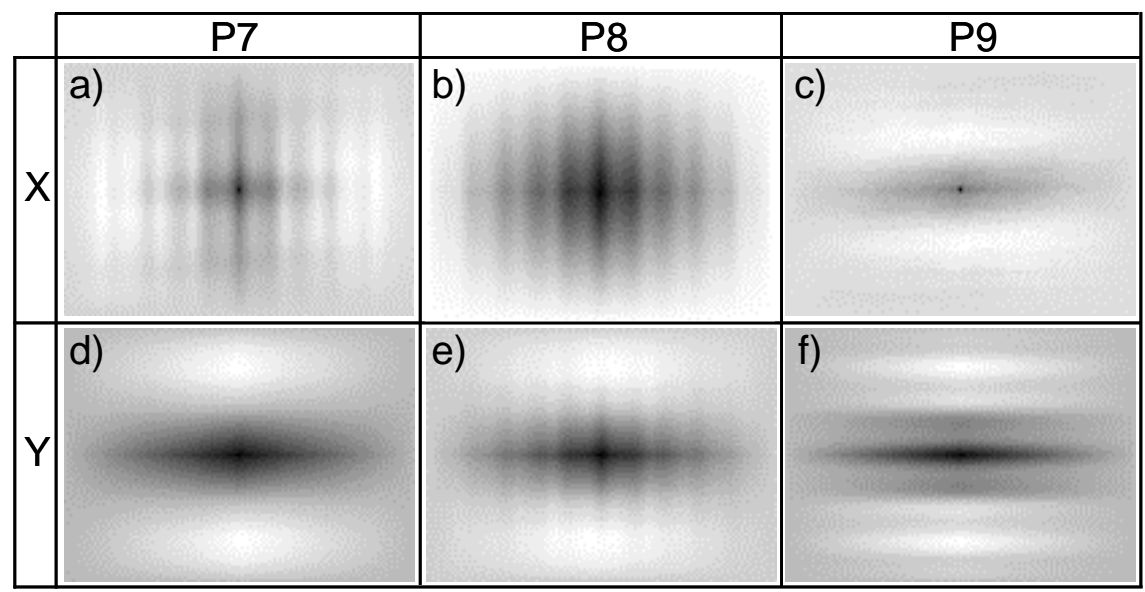

Figure 6: Images of self-correlation corresponding to images of displacement in $x$ (top) and in $y$ (bottom) directions from conditions P7, P8 and P9 shown in Figure 4.

appearance of a pattern in both $x$ and $y$ directions. The standard deviation of each image $\left(S D^{s c}\right)$ has been calculated for both directions $x$ and $y\left(S D_{x}^{s c}\right.$ and $\left.S D_{y}{ }^{s c}\right)$ in order to distinguish the different self-correlation images. For instance, conditions P5 and P6 have similar values of parameters $S D_{x}$ and $R_{x}$, although $S D_{x}{ }^{\text {sc }}$ values are very different. $S D_{x}{ }^{\text {sc }}$ is much larger in $\mathrm{P} 5$ than in P6, which indicates higher degree of order in P5 (cf. Table 2 and Fig. 5).

Thus, a random distribution can be distinguished from an ordered one by using parameter $S D^{s c}$. Nevertheless, besides the information relative to order, there is some influence of the values of displacement themselves. Thus, condition P6 and P9 have a similar value of $R_{x}$ characteristic of their random distribution, but different values of $S D_{x}{ }^{S C}$ are obtained in relation with their values of $S D_{x}$. In fact, a similar behaviour is found for P7 and P9 for $y$ displacements (cf. Table 2). Thus, we can say that not only the presence of ordered patterns influences the value of $S D^{s c}$, but also the level of noise in the original image.

Nevertheless, low values of $S D^{s c}$ typically indicate good situations, which involve random distributions with low level of noise. In case of $x$ displacements, the lowest value of $S D^{s c}$ is obtained in P9, which shows a neutral value of $R_{x}$ and the minimum value of $S D_{x}$. For $y$ displacements, $\mathrm{P} 2$ seems to be the best one, with neutral value of $R_{y}$ and the lowest value of $S D_{y}$. P6 is also good, with low ordering from $R_{y}$ and very low $S D_{y}$.

\section{Conclusions}

A proper measurement of internal stress in solids using combination of SEM imaging, focused ion beam milling and digital image correlation technique requires images collected by SEM at conditions that minimize and randomize 
displacement noise introduced by scanning electron microscope itself. In this work we proposed few methods for testing of SEM imaging conditions and selection those which do not influence the quality internal stress measurement substantially.

The stability of a Philips XL30 ESEM scanning electron microscope images has been evaluated for different imaging conditions by calculating the standard deviation of the displacements between two subsequent images from the same object. In general, this microscope is quite unstable, and not very useful for applications involving DIC. Nevertheless, the roles of magnification and image resolutions have been described. It is necessary to operate at a magnification high enough to reach a reasonably small pixel size.

In addition, the degree of randomness of the noise has been estimated by the definition of appropriate statistical parameters. Parameter ratio $R$ between the average of the standard deviation $(\overline{S D})$ of the displacements in image columns ( $\left.\overline{S D}^{\text {cols }}\right)$ and lines $\left(\overline{S D}_{\text {rows }}\right)$ for both horizontal and vertical displacements is sensitive to ordering in rows and columns. Also the use of self-correlation images has proven to highlight the presence of any ordering in the testing images. The standard deviation of such images is a valuable tool to quantify the degree of order, and to distinguish among different situations. Nevertheless, this parameter has to be used with care, since it is not only sensitive to the degree of order but also to the amount of noise in the images of displacement.

\section{Acknowledgement}

This research was carried out under project number M61.7.10415 in the framework of the Research Program of the Materials innovation institute, M2i (www.m2i.nl).

\section{References}

[1] P.J. Withers and H.K.D.H. Bhadeshia, "Residual stress part 2 - Nature and origins,” Materials Science and Technology, vol. 17, no. 4, pp. 366-375, 2001.

[2] R. Prummer and H. Pfeiffervollmar, "A Method for X-Ray Stress-Analysis of Thermochemically Treated Materials,” Advances in X-Ray Analysis, vol. 26, pp. 225-231, 1983.

[3] A.L. Shull and F. Spaepen, "Measurements of stress during vapor deposition of copper and silver thin films and multilayers,” J. Appl. Phys., vol. 80, no. 11, pp. 6243-6256, Dec. 1996.

[4] K.D. Bouzakis, G. Skordaris, S. Hadjiyiannis, A. Asimakopoulos, J. Mirisidis, N. Michailidis, G. Erkens, R. Cremer, F. Klocke, and M. Kleinjans, "A nanoindentation based determination of internal stress alterations in PVD films and their cemented carbides substrates induced by recoating procedures and their effect on the cutting performance," Thin Solid Films, vol. 447, pp. 264-271, Jan. 2004. 
[5] D.V. Nelson, "Residual Stress Determination by Hole Drilling Combined with Optical Methods," Exp. Mech., vol. 50, no. 2, pp. 145-158, Feb. 2010.

[6] Z.L. Kahn-Jetter and T.C. Chu, "Three-dimensional displacement measurements using digital image correlation and photogrammic analysis," Experimental Mechanics, vol. 30, no. 1, pp. 10-16, 1990.

[7] M.A. Sutton, J.J. Orteu, and H.W. Schreier, Image Correlation for Shape, Motion and Deformation Measurements - Basic Concepts, Theory and Applications. New York, 2009.

[8] K.J. Kang, N. Yao, M.Y. He, and A.G. Evans, "A method for in situ measurement of the residual stress in thin films by using the focused ion beam,” Thin Solid Films, vol. 443, no. 1-2, pp. 71-77, October 2003.

[9] N. Sabate, D. Vogel, A. Gollhardt, J. Keller, C. Cane, I. Gracia, J.R. Morante, and B. Michel, "Residual Stress Measurement on a MEMS Structure With High-Spatial Resolution,” J. Microelectromech. Syst., vol. 16, no. 2, pp. 365-372, Apr. 2007.

[10] B. Winiarski, R.M. Langford, J. Tian, Y. Yokoyama, P.K. Liaw, and P. J. Withers, "Mapping residual stress distributions at the micron scale in amorphous materials," Metallurgical and Materials Transactions A: Physical Metallurgy and Materials Science, vol. 41, no. 7, pp. 1743-1751, 2010.

[11] J.Q. Da Fonseca, P.M. Mummery, and P.J. Withers, "Full-field strain mapping by optical correlation of micrographs acquired during deformation,” J. Microsc., vol. 218, pp. 9-21, Apr. 2005.

[12] J.C. Russ, The Image Processing Handbook, Sixth Edition. CRC Press, 2011. 